\title{
Experiencias docentes y su proyección en la identidad profesional: el caso de las maestras de Santa Marta y su entorno $^{1}$
}

\author{
Teaching experiences and their projection in the professional identity: a \\ case-study of teachers in Santa Marta and their environment
}

Experiências docentes e sua projeção na identidade profissional: o caso das professoras de Santa Marta e seu entorno

María Concepción Domínguez Garrido²

Universidad Nacional de Educación a Distancia-UNED (España)

Adiela Ruiz Cabezas ${ }^{3}$

Universidad Nacional de Educación a Distancia-UNED (España)

Antonio Medina Rivilla ${ }^{4}$

Universidad Nacional de Educación a Distancia-UNED (España)

Grupo de investigación HISULA

Recepción: 23/05/2016

Evaluación: 28/03/2017

Aceptación: 24/08/2017

Artículo de Investigación

DOI: https://doi.org/10.19053/01227238.7555

\section{RESUMEN}

Este artículo presenta los resultados de Riohacha (Colombia) con un grupo de una investigación llevada a cabo en Santa Marta y el corregimiento de Juan y Mediomaestras afrodescendientes. El principal objetivo fue identificar las experiencias

1 Este artículo se relaciona con los proyectos de investigación: Historias de vida. Maestras de escuela de mediados del siglo XX y del siglo XI en la zona Cundiboyacense SGI: 980. UPTC, La Universidad en la capacitación de las maestras rurales en Colombia y Guatemala SGI 1034 UPTC y Formación de educadores en las Escuelas Normales Superiores. Convenio UPTC. 2010-2017. SGI. 2192 Dirección de Investigaciones Universidad Pedagógica y Tecnológica de Colombia. Lidera Dra. Diana Elvira Soto Arango.

2 Doctora en Educación. Profesora Universidad Nacional de Educación a Distancia-UNED (España). Grupo de Investigación "Desarrollo profesional: formación e Innovación Educativa e Intercultural y Diseño de Medios" (Ref.: 125-UNED). Email: cdominguez@edu.uned.es.

3 Doctora en Educación. Profesora-Tutora Universidad Nacional de Educación a Distancia-UNED (España). Email: adiruiz@madrid. uned.es.

4 Doctor en Educación. Profesor Universidad Nacional de Educación a Distancia-UNED (España). Grupo de Investigación “Desarrollo profesional: formación e Innovación Educativa e Intercultural y Diseño de Medios” (Ref.: 125-UNED). Email: amedina@edu.uned. es. 
formativas que han marcado su estilo docente y las ha llevado a compartir una línea de desarrollo profesional, apoyada en el discurso narrativo y de implicación para dar respuesta a nuevos modelos de investigación y desarrollo profesional. A través de cuestionarios y entrevistas se recogieron las narrativas de 10 maestras, quienes respondieron a preguntas sobre sus experiencias formativas más significativas, teniendo en cuenta aspectos como: dónde, cuándo, por qué, para qué, con quién, qué, cómo, y sobre el impacto que ha tenido la experiencia docente en su desarrollo profesional. Se ha utilizado la metodología narrativa dado que el profesorado descubre en la identificación y análisis de las experiencias profesionales más innovadoras y relevantes que han vivido, las bases para aprender de su profesión y tomar decisiones para mejorar su identidad con ella. Los hallazgos derivados de esta investigación explicitan un camino de afianzamiento del pensamiento y actuaciones formativas, de las maestras implicadas, concretadas en: contextos escolares y familiares, finalidades para alcanzar el desarrollo profesional, desempeños en la práctica docente, escenarios de pluralidad y de encuentros de las culturas, así como en tiempos y cronología vivida.

Palabras clave: historias de vida; narrativas; interacción cultural; desarrollo profesional; Revista Historia de la Educación Latinoamericana.

\section{ABSTRACT}

This article presents the results of a research carried out in Santa Marta and in the rural area of Juan y Medio-Riohacha (Colombia) with a group of Afrodescendant teachers. The main objective was to identify the formative experiences that have left a mark on their teaching style and have led them to share a line of professional development, supported by a narrative and involvement discourse; in order to respond to new models of research and professional development. Through questionnaires and interviews, the narratives of 10 teachers were collected, they answered questions about their most significant training experiences, taking into account aspects such as: where, when, why, what for, with whom, what, how, and about the impact of the teaching experience on their professional development. The narrative methodology was used since it helps teachers to discover in the identification and analysis of their most innovative and relevant professional experiences, the bases to learn from their profession and make decisions to improve their identity with it. The findings derived from this research reveal a path of consolidation of thought, and formative actions framed in: school and family contexts, goals to achieve professional development, performance in teaching practice, scenarios of plurality and meetings of cultures, as well as a teaching timeline.

Keywords: life stories; narratives; cultural interaction; professional development; Journal History of Latin American Education.

\section{RESUMO}

Este artigo apresenta os resultados de uma pesquisa realizada em Santa Marta e na jurisdição de Juan e Meio-Riohacha (Colômbia) com um grupo de professoras afrodescendentes. O principal objetivo foi identificar as experiências formativas que marcaram seu estilo docente e as levaram a compartilhar uma linha desenvolvimento profissional, apoiada no discurso narrativo e de implicação resposta a novos modelos de pesquisa e desenvolvimento profissional. Por meio de questionários e entrevistas se recorreu às narrativas de 10 professoras, que responderam a perguntas sobre suas experiências formativas mais significativas, tendo em conta aspectos como: onde, quando, por que, para que, com quem, o que, como e sobre o impacto que a experiência docente teve 
em seu desenvolvimento profissional. Foi utilizada a metodologia narrativa considerando que o professorado descobre na identificação e análise das experiências profissionais mais inovadoras e relevantes que viveram, as bases para aprender sua profissão e tomar decisões para melhorar sua identidade com ela. As descobertas desta pesquisa explicitam um caminho de segurança do pensamento e atuações formativas, das professoras implicadas, sustentadas em: contextos escolares e familiares, finalidades para alcançar o desenvolvimento profissional, desempenhos na prática docente, cenários de pluralidade e de encontros das culturas, assim como em tempos e cronologias vividas.

Palavras-chave: histórias de vida, narrativas; interação cultural; desenvolvimento profissional; Revista História da Educação Latino-americana.

\section{Contextualización: Idioma Maya Ch'orti'} Contextualizador: Lucas López de Rosa Ministerio de Educación de Guatemala

\section{B'IJK TZ'IJB'}

E tz'ijb'ayaj ira ucheksu tama e sajkmayaj xe' uche e Santa Marta yi e ajk'anpa'r Juan yi Ujam -Riohacha ( Colombia) taka inte' morwa'r te ajkanseyajob' ekpak'ab'ob'. Ch'enpa e'ra twa' asajkna una'tanyaj kochwa' apatna e pak'ab', takarseb'ir taka e cheksuyaj twa'tix ajk'unpa tuk'a e topop te sajkmayaj twa' inte' k'anpa'rir kanseyaj. Umen taka e sajkmayaji yi e ub'esyaj xe' tajwina taka inla'inte' ajkanseyaj, chyob', xe' uyajk'ob' e ub'esyaj kochwa' una'tanyaj xe' uk'ojkob', tya' ch'ajma e ub'esyaj kochwa': tya', tuk'a, tuk'at, tuk'a twa', chi takar, yi tuk'at ak'anpa una'tanyaj e ajkanseyaj

Uk'anpes e sajkmayaj te k'ajtmayaj umen kochwa' e ajkanseyaj ak'otoy utajwi yi uwira e na'tanyaj te pak'ab' $x e^{\prime}$ inb'utz yi ak'anpa kochwa' una'ta, k'otoy utajwi tama e patna'r xe' uche twa'tix o'stanpa u'tir kochwa' ja' xir.

$\mathrm{Xe}^{\prime}$ utajwi tama e sajkmayaj ira ucheksu e b'i'r twa' atajwintz'a inb'utz b'ijsyaj kochwa' ujajpi, taka e ajkanseyaj xe' utajwyob' xe' ak'a' pa taka e ototir kanseyaj yi tatob', uk'a' pa'r yi e takarsaj ajkanwa'r, twa' apatna te ejtz'una'r kanseyajir, ucheksu e me'yrir pak'ab' tya' utajwi e pak'ab' , b'an twa' e numerir yi tuk'a tuk'a anumuy.

Tz'ijb' ak'anpa: Utz'ijb' e b'ixirar, $k^{\prime} a j t m a y a j ; \quad m o r w a^{\prime} r$ pak'ab'; takseyaj patna'rir; Jun twa'r unumer tama e Kanseyajir te Rumob'.

\section{INTRODUCCIÓN}

La elección de prácticas docentes significativas para el profesorado y el proceso de convertirlas en casos relevantes, de los que cada docente ha de aprender y compartir como núcleo destacado de la toma de decisiones en los contextos inciertos del aula, se ha valorado como un foco sustantivo para avanzar en nuevas actuaciones docentes.
El valor de las narrativas (historias de vida, autobiografías) y diversidad de experiencias formativas vividas y compartidas por las maestras que han asumido la profesión como el camino más valioso para propiciar la educación integral de las personas, se ha constatado en diversas investigaciones. Así, Cañón y Cols $(2016)^{5}$, en una investigación realizada

5 Lilia Cañón Flórez, Myriam Esperanza Monroy González y Javier Ricardo Salcedo Casallas. "Experiencia pedagógica y voz 
a partir de las narrativas de una maestra afro indagan sobre la cotidianidad y la interacción para visibilizar el entramado donde se tejen las prácticas y saberes escolares que hacen posible el fortalecimiento de las relaciones multiculturales.

Los resultados de la investigación realizada por Soto Arango y Cols (2016) ${ }^{6}$, en Malabo (Guinea Ecuatorial) sobre historias de vida a un grupo de maestras, utilizando la técnica de narrative inquiry, muestran que el grupo de participantes presentan diversas actuaciones formativas, desde la época colonial hasta nuestros días, que han tenido un gran impacto en su vida profesional y personal.

Destacamos el trabajo realizado por el grupo de investigación HISULA, de la Universidad Pedagógica y Tecnológica de Colombia, dirigido por la Doctora Diana Soto Arango, sobre Historias de vida de maestras afrodescendientes, cuyos trabajos han sido publicados en un número especial de la Revista Historia de la Educación Latinoamericana ${ }^{7}$, como un referente de la importancia de este enfoque investigativo para indagar sobre las prácticas docentes, experiencias formativas, relaciones entre culturas, etc.

Por otro lado, los trabajos de Goodson y Cols (2012) ${ }^{8}$, Medina, (1996) ${ }^{9}$, Medina y Domínguez $(2015)^{10}$, destacan que los docentes al seleccionar justificadamente aquellas experiencias formativas que les han marcado como profesionales, asumen que las prácticas son auténticos procesos para potenciar su desarrollo profesional y consideran las reflexiones y narrativas emergentes de tales actuaciones formativas, una base liberadora y de plena consolidación de su línea de innovación docente.

Entre las líneas y modalidades formativas más relevantes que las maestras han de asumir en esta investigación, destacan: "Identificación, narración-exposición, análisis y toma de conciencia" de algunas experiencias y prácticas educativas vividas en intensidad con estudiantes, familias y colegas en diversas fechas, constituyendo un referente esencial para dar respuesta al reto de una educación centrada en las auténticas necesidades de las personas que se forman.

La acción formadora es un proceso de intensa búsqueda de alguna experiencia relevante focalizada en lo más destacado de la práctica educativa, por su sentido holístico y transversal. La tarea asumida se centra en una revisión y puesta a punto del recuerdo diferido de aquellas formas de mejora y de reconocimiento de la acción docente en toda su amplitud, al requerir de una revisión amplia de las más destacadas experiencias profesionales desempeñadas en la vida educativa de las participantes.

La identificación implica un proceso personal y compartido para poder determinar de las numerosas acciones desempeñadas, alguna que no solo sobresale

multicultural: Pasos y palabras en historia de vida de maestra afrodescendiente", Revista Historia de la Educación Latinoamericana 18, No. 27 (2016):167-190.

6 Diana Elvira Soto Arango, Ma Teresa Avoro Nguema Ebana, Adiela Ruiz Cabezas y Antonio Medina Rivilla. "Formación de docentes en Guinea Ecuatorial. Historias de vida de maestras en prospectiva al 2020", Revista Historia de la Educación Latinoamericana Vol. 18, No. 27 (2016): 67-94.

7 Para más información consultar: Revista Historia de la educación Latinoamericana. Vol. 18, No. 27 (2016)

8 Ivor Goodson et al (Eds.). Explorations in narrative research. (Rotterdam: Sense Publishers, 2012).

9 Antonio Medina Rivilla. "La biografía perspectiva de la formación del profesorado: limitaciones y posibilidades". En Historias de vida e investigación etnográfica, eds. Emilio López-Barajas Zayas y Magdalena Montoya Sáenz (Madrid: UNED, 1996).

10 Antonio medina Rivilla y Ma Concepción Domínguez Garrido “Modelos didáctico-tecnológicos para la innovación educativa”. En Recursos tecnológicos en contextos educativos, eds. Ma. Luz Cacheiro, Cristina Sánchez y Jesús Manuel González (Madrid: UNED, 2015). 
de entre las numerosas prácticas experimentadas, si no que se ha de centrar en la que a su juicio es altamente valorada por su impacto en el avance profesional y en las restantes actividades docentes.

La experiencia identificada por su gran potencialidad formativa y de impacto en el aprendizaje como maestra, ha de cumplir:

- Amplitud de vivencias y percepción de la pluralidad de prácticas docentes.

- Idiografía de las acciones elegidas y generadas en su práctica profesional.

- Armonía entre la innovación docente y reconocimiento de lo más valioso de su vida de educadora.

- Pertinencia de las prácticas elegidas para dar respuesta a los nuevos retos de los estudiantes, familias y sociedad.

- Explicación del modelo educativo que subyace al trabajo realizado en los ambientes de aprendizaje.

La redacción y explicitación de un título claroy preciso de la práctica profesional, evidencia la idea central que subyace al conjunto de las decisiones propias de la experiencia que caracteriza este proceso.

Se entiende que las maestras al exponer el título más expresivo y clarificador de lo que espera compartir con las colegas y la comunidad científica, lleva a cabo una tarea metacognitiva que influye creativamente en su propia capacitación y en la mejor interpretación de las tipologías de prácticas que han de asumir y compartir, para lograr un escenario de colaboración y de sistematización de este proceso reflexivo.

Se ha propuesto que esta primera fase de identificación y nominación de la experiencia sea completada por la "narrativa" de la misma, facilitando el auténtico conocimiento de las claves en y desde los que vive cada maestra la acción que le ha transformado desde las prácticas, y que al retomarla, reflexionarla y redactarla con máxima meticulosidad, ofrece una auténtica transformación y mejora integral de la acción docente, delimitándose como un valioso camino y proceso de desarrollo profesional.

\section{Preguntas clave en las narrativas}

Se han consolidado algunos perfiles orientadores de narrativas profesionales que responden a una presentación de las mismas, apoyadas en preguntas, entendidas en un estilo interrogativo-reflexivo que sitúan a cada maestra como la mejor protagonista de su propia historia. Así, hemos de apoyar a las maestras para que orienten sus reflexiones en torno a las cuestiones que mejor formalicen y caractericen sus relatos práctico-expresivos, de tal manera que se les invita a responder a las preguntas más características de los procesos educativos que, a nuestro juicio, faciliten nuevas respuestas clarificadoras a los interrogantes:

- ¿Dónde tuvo lugar la experiencia formativa-transformadora? (visión glocalizadora) escenarios virtuales.

- ¿Cuándo se llevó a cabo y en qué línea y ciclo de vida de la maestra tuvo lugar?

- ¿Por qué?, ¿Qué significa para la maestra esta experiencia? 
- ¿Para qué? La experiencia tiene y cumple una funcionalidad singular. ¿Cuál? ¿Qué se espera alcanzar?

- ¿Con quién? En toda su amplitud: estudiantes, familias, comunidades, culturas, sociedad, escenarios en evolución, etc.

- ¿Qué? Se focaliza en el contenido más representativo de la experiencia elegida, que armonice los aspectos más pertinentes de la práctica docente y explicite lo valioso de ella.

- ¿Cómo? La maestra ha de expresar, qué métodos didácticos han caracterizado esta experiencia formativa y qué actividades se han llevado a cabo para realizar la práctica docente que se narra.

- ¿Qué impacto ha tenido en su desarrollo profesional?

Esta repercusión es esencial dado que cada maestra ha de explicitar aquella visión que ha destacado y cómo este proceso de identificar, narrar y proyectar la práctica más relevante, ha de sintetizarse en descubrir en cada persona la incidencia percibida y las mejoras esperadas.

Estas cuestiones han de ser tenidas en cuenta por cada maestra que se proponga aprender de su vida profesional y desee convertir su práctica innovadora en objeto de reflexión y aprendizaje profesional.

El proceso de emerger alguna actuación profesional previa, se ha de concretar en la explicación de algunos aspectos innovadores que la práctica ha tenido para cada maestra, sirviendo de escenario singular para entender y compartir cada una de las decisiones que han hecho factible que tal acción docente, sea considerada relevante y de intensa repercusión personal e institucional. Al expresar el relato de la actuación elegida, las maestras se sitúan ante su propio conocimiento como profesionales y se configura el verdadero escenario que les facilita llegar a ser responsables de su historia docente, asumiendo ingentes posibilidades de ser las protagonistas de su línea de avance y mejora como educadoras en un mundo en complejidad ascendente.

¿Cómo lograr la complementariedad entre narrar con rigor una experiencia formativa y consolidar un proceso de investigación e innovación docente?

Las narrativas son el marco en el que se da significado a las experiencias, las hacen significativas y le dan sentido (Bochner, et al 2003) ${ }^{11}$, en este sentido, la implicación de las maestras en procesos narrativos centrados en la práctica profesional, ha de servir como base para que cada una descubra en tal práctica, los valores de su opción personal y una base para avanzar y descubrir el auténtico significado y transformación de lo que las ha marcado como protagonistas de la actividad docente. En definitiva, se trata de que puedan situar esta reflexión y armonización de la práctica docente, en un acto de investigación e innovación consustanciales a los programas de desarrollo profesional de los docentes.

La narración de cada maestra y su compromiso con una rigurosa presentación de los logros más relevantes alcanzados en la práctica educativa elegida, se

11 Arthur Bochner, C. Ellis, I. Sava, K. Nuutinen, M. Mäkelä, M.E. Ylönen \& S.D.Sclater, "The arts and narrative research: Art as inquiry", Qualitative inquiry, 9 No. 4 (2003): 505-624. 
evidencian en una doble proyección: el avance profesional de la maestra que sintetiza y emerge la práctica vivida, y el acompañamiento a cada estudiante, familia, escuela y comunidad, quienes constituyen los co-agentes y contexto en el que ha construido este estilo de tomar decisiones, avanzar como investigadora y aprender a consolidar un modelo de innovación.

Medina y Medina, $(2015)^{12}$, ha propuesto algunos modelos para afianzar la innovación de la educación y de la docencia, destacando el de Mejora de la escuela (Leithwood et al 2006 ${ }^{13}$, Leithwood y Hallinguer 2012) ${ }^{14}$, sociocomunicativo (Medina, $2015^{15}$ y Cazden, 2010) ${ }^{16}$, ampliados por un marco singular, que corresponde a un modelo de entender y mejorar las prácticas educativas argumentadas por cada maestra en la acción formativa, desde una visión y génesis afrocolombiana, dado que la construcción de las experiencias está ligada al modo de percibir y de comprometerse en las diversas acciones formativas, singularmente las modalidades de cultura que emergen y se consolidan en multitud de episodios (Lima y Michaltchuk, (2015) ${ }^{17}$.

En síntesis, consideramos que el análisis de las experiencias formativas previas y su impacto en la mejora del desarrollo profesional son importantes para impulsar el trabajo y prácticas del profesorado (Huber, 2010, $2014^{19}$; Medina 201420; Medina y Domínguez, 1995²1); así, Denzin (2014, 422) ${ }^{22}$, enfatiza que "la etnografía no es una práctica inocente, la investigación de la práctica es performadora, pedagógica y política. A través de los escritos y de las conversaciones actuamos y compartimos nuestros mundos, dado que la pedagogía es siempre moral y política".

12 María Medina Domínguez y Antonio Medina Rivilla. "Modelos didácticos y métodos para el desarrollo del conocimiento y la cultura innovadora de las instituciones educativas", En Innovación de la Educación y de la Docencia 2 da. Ed., coord. Antonio Medina Rivilla (Madrid: Ramón Areces, 2015), 29-63.

13 Kennet A. Leithwood, Doris Jantzi and Charrin McElheron-Hopkins. "The development and testing of a school improvement model ", School effectiveness and school improvement 17, No. 4 (2006): 441-464.

14 Kenneth A. Leithwood and Philip Hallinger, eds. Second international handbook of educational leadership and administration (Springer Science \& Business Media, 2012).

15 Antonio Medina Rivilla, coord. Innovación de la Educación y de la Docencia 2da. Ed. (Madrid: Ramón Areces, 2015).

16 Courtney B. Cazden "Las aulas como espacios híbridos para el encuentro de las mentes." En Aprendizaje y contexto: contribuciones para un debate, ed. Nora Elichiry Buenos Aires: Ediciones Manantial, 2010).

17 José Rubén Lima Jardilino, e Ivanna Elena Michaltchuk. "La maestra en la escuela rural itinerante: Una experiencia de educación rural en la región sur de Brasil”. En La maestra rural en Iberoamérica, eds. Diana Elvira Soto, Justo Cuño y Oscar Hugo López, (Tunja: Ed. FUDESA, 2015), 271-299.

18 Huber Huber. "Aprendizaje activo y metodologías educativas", Revista de Educación, Número extraordinario (2008): 59-81.

19 Gunter Huber. "Investigación en la comunicación didáctica”. En Las Fronteras de la Investigación Didáctica eds. Antonio Medina, Agustín de la Herrán y Ma Concepción Domínguez. (Madrid: Editorial UNED, eBook 2014), 73-114.

20 Antonio Medina Rivilla. "La investigación como base del conocimiento didáctico y de la innovación de la enseñanza". En Las Fronteras de la Investigación Didáctica, eds. Antonio Medina, Agustín de la Herrán y Mª Concepción Domínguez. (Madrid: Editorial UNED, eBook 2014), 23-72.

21 Antonio Medina Rivilla y M ${ }^{a}$ Concepción Domínguez Garrido. "Constructing. teacher's practical knowledge through analysis of Geography Teacher's Discourse". En Experimental Research on Teaching and Learning. Eds. Olechowski y Khan-Srik (Frankfurt: Peter Lang 1995), 95-117.

22 Norman Denzin. Interpretative autoethnography. (California: Thousand Oaks, 2014). 
En esta línea, Chase $(2011,430)^{23}$, expresa que las narrativas cuestionadoras son un campo en el que se construye al profundizar y preguntar acerca de los colegas, las conversaciones y las comunidades, al sugerir que se combinan entrevistas con observación etnográfica, fotografías y escritos autobiográficos, mapas, sistemas de información geográfica y análisis de contenido de documentos.

\section{Diseño metodológico}

La investigación realizada se inserta en la modalidad de investigación narrativa de Chase $\left(2011^{24}\right.$, Connelly y Clandinin, $\left.1990^{25}, 2006^{26}\right)$ y se completa con el método de síntesis de contenido y comprensión de la singularidad de los discursos en contextos en evolución que caracteriza la investigación de carácter emergente y presidida por los objetivos acordados entre docentes e investigadores, conscientes del papel de la reflexión personal en el desarrollo de la investigación.

Se explicita un objetivo general compartido entre investigadores:

- Identificar las experiencias formativas que han marcado su estilo docente y compartir una línea de desarrollo profesional, apoyada en el discurso narrativo y de implicación de las maestras para dar respuesta a nuevos modelos de investigación y desarrollo profesional.

Justificamos la narrativa interrogativa como una metodología que se aplica mediante el empleo de un proceso interrogatorio, que estimule a las maestras a emerger los aspectos nucleares que caracterizan los procesos de enseñanzaaprendizaje y la evolución de la práctica profesional. La metodología narrativa se configura como la aportación pertinente para presentar los procesos vividos por cada maestra y retroinformarle para que descubra los significados explícitos e implícitos de las prácticas educativas desempeñadas.

Las narrativas orales y escritas se recogieron durante los años 2015 y 2016; así, se invita a las maestras a identificar la experiencia formativa más valiosa y de mayor impacto en su desarrollo como docente, descubriendo las respuestas pertinentes a los siguientes aspectos que hemos referido anteriormente (Dónde, cuándo, por qué, para qué, cómo, etc.).

La investigación en torno a las narrativas presentadas se caracteriza por el énfasis cualitativo y la interpretación de los elementos expresados en los relatos que sintetizan la fuerza e importancia de lo expresado, así como el impacto de las experiencias vividas desde prácticas reflexivas y relevantes que han marcado el estilo de percibir y de experimentar los trabajos desempeñados en las escuelas y en las comunidades, tanto urbanas (barrios peculiares y maestras

23 Susan E. Chase. "Narrative Inquiry. Still a field in the making". En The Sage Handbook of Qualitative Research, eds. Norman Denzin y Yvonne S. Lincoln. (California: Thousand Oaks, 2011), 421-434.

24 Ibid.

25 F.M. Connelly y D. J. Clandinin, "Stories of experience and narrative inquiry", Educational Researcher, 19 No. 5 (1990): 2-14.

26 F.M. Connelly y D. J. Clandinin, "Narrative inquiry", en Complementary methods for research in education, eds. JL Green y G. Camilli, (PB Elmore Eds., 2006): 477-487. 
afrocolombianas) como rurales. Consideramos que, desde las experiencias narradas por las participantes, han emergido el significado y potencialidad para avanzar en la propia práctica, al seleccionar aquella más transformadora vivida por cada profesional/persona.

Una vez transcritas las entrevistas, los análisis de contenido de las narrativas se han completado con la triangulación del juicio de los investigadores, completados con el estudio intenso de los datos mediante la aplicación del software Atlas ti, que ha facilitado la presentación y categorización de los hallazgos más valiosos emergidos de los datos.

Se ha intensificado el análisis de las narrativas y dialogado con varias maestras implicadas en la investigación, empleando un recurso interactivo como el WhatsApp, para ampliar la pertinencia de los datos y validar aún más las categorías emergentes (Creswell, 200727, 2012 28 ). Todo ello dio lugar a la aparición de nuevas pistas, la coherencia de los datos del programa y la apertura a nuevas interpretaciones, que fueron cotejadas entre investigadores y maestras, avanzando en la calidad de los modelos de formación desde la práctica reflexiva y colaborativa.

\section{Análisis y resultados}

\section{Contexto}

Los escenarios de las maestras implicadas tienen una intensa influencia en la construcción de las modalidades y significado de las prácticas vividas y su valoración para incidir en la mejora de cada persona participante en este proceso, así, describimos e interpelamos los ambientes reales y virtuales vividos por las maestras en el Caribe colombiano.

Imagen 1. Contexto geográfico de las maestras afrocolombianas implicadas en la investigación.

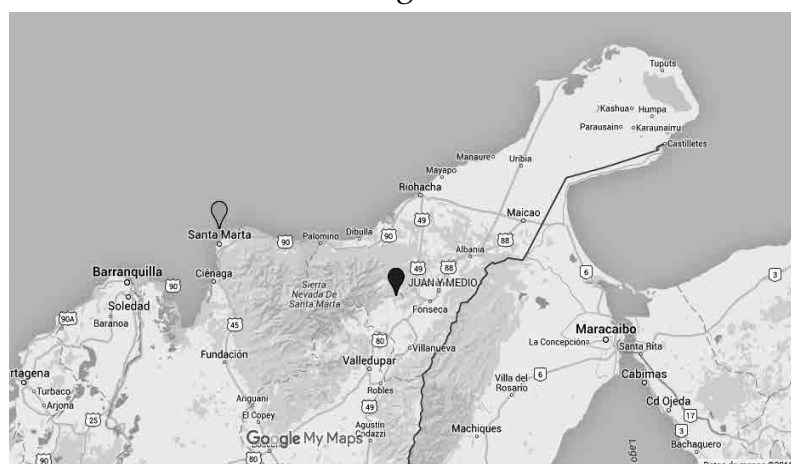

Fuente: Elaboración propia con la aplicación Google Maps.

27 J.W. Creswell, Qualitative inquiry \& research design: Choosing among five approaches (Thousand Oaks, US: SAGE, 2007).

28 J. W Creswell, (2012). Educational research: Planning, conducting and evaluating quantitative and qualitative research (Boston, US: Pearson, 2012). 
Las maestras participantes en la producción de narrativas innovadoras que han enriquecido la línea de desarrollo profesional de las docentes afrocolombianas, de gran incidencia en la visión del encuentro y diálogo entre culturas, viven y trabajan en el Caribe Colombiano en la ciudad de Santa Marta Departamento del Magdalena (contexto urbano) y en el corregimiento de Juan y Medio (contexto rural) perteneciente al Municipio de Riohacha en el Departamento de la Guajira.

Presentamos los datos más relevantes referentes al contexto en los que trabajan las maestras participantes en la siguiente matriz:

Tabla 1. Contexto en el que trabajan las maestras afrocolombianas implicadas en la investigación.

\begin{tabular}{|c|c|c|c|}
\hline Maestra & $\begin{array}{l}\text { Espacio socio- } \\
\text { geográfico }\end{array}$ & Institución & Comunidad \\
\hline M. R. & \begin{tabular}{|l|} 
Santa Marta. \\
Etnoeducación Afro- \\
Básica Primaria- \\
\end{tabular} & $\begin{array}{l}\text { Institución Educativa } \\
\text { Distrital } 20 \text { de Octubre }\end{array}$ & $\begin{array}{l}\text { Afrodescendientes, } \\
\text { mestizos. }\end{array}$ \\
\hline R. R. & $\begin{array}{l}\text { Santa Marta. Barrio } \\
\text { en situación de } \\
\text { marginalidad }\end{array}$ & $\begin{array}{l}\text { Institución Educativa } \\
\text { Distrital El Parque }\end{array}$ & $\begin{array}{l}\text { Afrodescendientes } \\
\text { mestizos. }\end{array}$ \\
\hline L. P. & $\begin{array}{l}\text { Santa Marta. Barrio } \\
\text { en situación de } \\
\text { marginalidad }\end{array}$ & $\begin{array}{l}\text { Institución Educativa } \\
\text { Distrital Cristo Rey }\end{array}$ & $\begin{array}{l}\text { Afrodescendientes, } \\
\text { mestizos. } \\
\text { Desplazados por la } \\
\text { violencia. }\end{array}$ \\
\hline J. C. & $\begin{array}{l}\text { Santa Marta. Barrio } \\
\text { en situación de } \\
\text { marginalidad } \\
\end{array}$ & $\begin{array}{l}\text { Institución Educativa } \\
\text { Distrital } 20 \text { de Octubre }\end{array}$ & $\begin{array}{l}\text { Afrodescendientes, } \\
\text { mestizos. }\end{array}$ \\
\hline A. R. S. & $\begin{array}{l}\text { Santa Marta Barrio } \\
\text { en situación de } \\
\text { marginalidad }\end{array}$ & $\begin{array}{l}\text { Institución Educativa } \\
\text { Distrital Cristo Rey }\end{array}$ & $\begin{array}{l}\text { Mestizos, } \\
\text { afrodescendientes }\end{array}$ \\
\hline V. M. & $\begin{array}{l}\text { Santa Marta. Barrio } \\
\text { en situación de } \\
\text { marginalidad }\end{array}$ & $\begin{array}{l}\text { Institución Educativa } \\
\text { Distrital } 20 \text { de Octubre }\end{array}$ & $\begin{array}{l}\text { Afrodescendientes, } \\
\text { mestizos. }\end{array}$ \\
\hline N.P. & $\begin{array}{l}\text { Santa Marta. Barrio } \\
\text { en situación de } \\
\text { marginalidad }\end{array}$ & $\begin{array}{l}\text { Institución Educativa } \\
\text { Distrital Cristo Rey }\end{array}$ & $\begin{array}{l}\text { Afrodescendientes, } \\
\text { mestizos. }\end{array}$ \\
\hline L.S. & $\begin{array}{l}\text { Juan y Medio, } \\
\text { Tomarrazón, La Palma }\end{array}$ & $\begin{array}{l}\text { Institución } \\
\text { Etnoeducativa Sierra } \\
\text { Nevada }\end{array}$ & $\begin{array}{l}\text { Afrodescendientes, } \\
\text { indígenas (Wayuu, } \\
\text { Wiwas, koguis y } \\
\text { arhuacos). }\end{array}$ \\
\hline I. S. & $\begin{array}{l}\text { Juan y Medio, } \\
\text { Tomarrazón, La Palma }\end{array}$ & $\begin{array}{l}\text { Institución } \\
\text { Etnoeducativa Sierra } \\
\text { Nevada }\end{array}$ & $\begin{array}{l}\text { Afrodescendientes, } \\
\text { indígenas (Wayuu, } \\
\text { Wiwas, koguis y } \\
\text { arhuacos). }\end{array}$ \\
\hline B. S. & $\begin{array}{l}\text { Juan y Medio, } \\
\text { Tomarrazón, La Palma }\end{array}$ & $\begin{array}{l}\text { Institución } \\
\text { Etnoeducativa Sierra } \\
\text { Nevada }\end{array}$ & $\begin{array}{l}\text { Afrodescendientes, } \\
\text { indígenas (Wayuu, } \\
\text { Wiwas, koguis y } \\
\text { arhuacos). }\end{array}$ \\
\hline
\end{tabular}

Fuente: Elaboración propia. 
Las maestras colombianas participantes en esta investigación tienen una media de 15 años de experiencia docente.

Las Instituciones educativas del contexto urbano (Santa Marta) en las que trabajan las maestras afrodescendientes, están situadas en barrios y zonas marginales, cuya población son afrodescendientes y mestizos, en muchos casos desplazados de sus lugares de origen por la situación de violencia. Como puede verse en la tabla anterior, los contextos en los que trabajan las docentes son zonas con una población en situación de alta vulnerabilidad social y precariedad económica, como lo expresan en las narrativas:

"Trabajo en Santa Marta en una Institución pública con estudiantes carentes de afecto, de atención en sus hogares y ha de trabajarse mucho la parte afectiva". ${ }^{29}$

"En el barrio donde está situada la Institución en la que trabajo hay mucha delincuencia, drogadicción, violencia intrafamiliar...". ${ }^{30}$

"Los estudiantes provienen de familias de escasos recursos económicos que desarrollan trabajos informales, pues durante muchos años dependieron del reciclaje realizado en el botadero de basura, que fue trasladado al sector de palangana, dejando a la población sin su fuente de trabajo y una alta contaminación ambiental"31.

El grupo de maestras que trabajan en el contexto rural pertenecen a la Institución Etnoeducativa Sierra Nevada que comprende tres Corregimientos: Tomarrazón, Juan y Medio y las Palmas, situados en las estribaciones de la Sierra Nevada de Santa Marta siendo la sede principal Juan y Medio, pero cuenta con 12 sedes más, distribuidas en diferentes veredas de la Sierra. Esta Institución Etnoeducativa es de carácter oficial, con un currículo flexible, democrático y participativo, ofrece sus servicios en la modalidad diurna, nocturna y sabatina en los niveles preescolar, básica primaria y básica secundaria; las edades de los estudiantes oscilan entre los cinco y sesenta años y pertenecen a diferentes grupos étnicos indígenas (wayuu,wiwa, kogui y arhuaca), mestizos y afrodescendientes en su gran mayoría.

29 Entrevista a R. R. Santa Marta, noviembre de 2015.

30 Narrativa de J. C. Santa Marta, abril de 2016.

31 Entrevista a M. R. Santa Marta, marzo de 2016. 


\section{Dónde}

Ilustración 1. Dónde fue realizada y cuál es la experiencia formativa de las maestras.

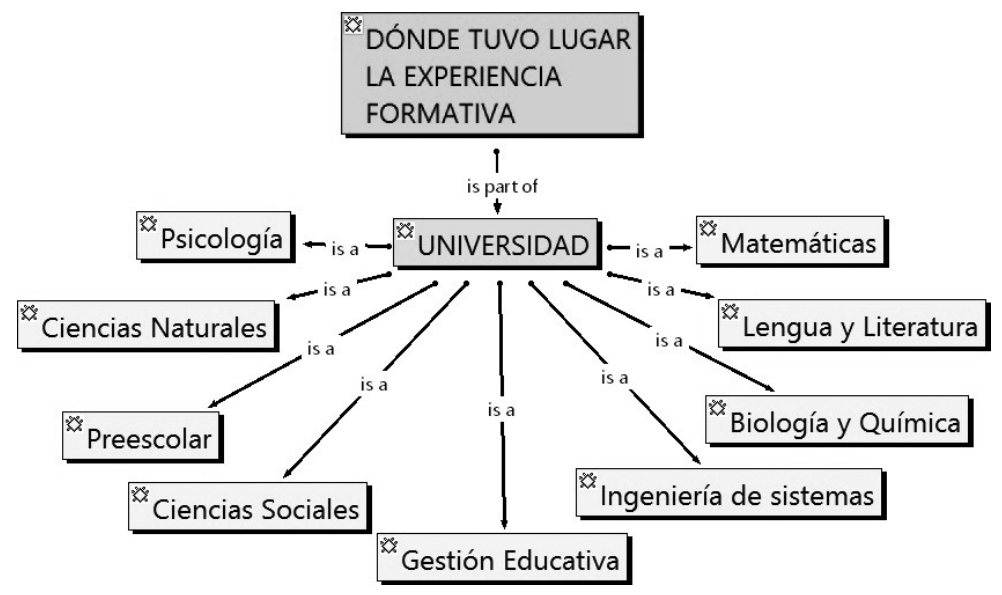

Fuente: elaboración propia mediante el programa Atlas ti.

Latotalidad delasmaestras son Licenciadas en diferentesáreas de conocimiento y la mayoría posee estudios de postgrado: Maestrías, Especializaciones y dos de ellas tienen Doctorado. La formación recibida les ha permitido desempeñarse en diferentes niveles educativos: preescolar, primaria, secundaria y universidad.

Con respecto a la motivación o vocación de ser maestras A. R., Licenciada en Ciencias Naturales, manifiesta:

"Durante las prácticas con estudiantes de primer semestre de la licenciatura me di cuenta de que esto era lo mío, además obtuve una buena evaluación por parte del docente de prácticas: la estudiante tiene el perfil y potencial de una buena docente". ${ }^{32}$

Para V. M., Ingeniera de Sistemas y profesora de Tecnología y Sistemas, fue transcendental la participación en la iglesia de su comunidad siendo muy joven: "Formar parte de un grupo de oración fue lo que impulsó mi vocación ${ }^{\prime \prime 3}$ y, más adelante en la etapa universitaria, destaca el apoyo y ejemplo de una de sus profesoras:

"...y la experiencia que tuve al estar en la clase de la profesora Clara Galindo en la universidad, donde me pude dar cuenta que tenía que tomar decisiones acerca de mi futuro como profesional y que definitivamente mi labor en este mundo era servir a los demás." 34

32 Narrativa de A. R. Serrano Santa Marta, mayo de 2016.

33 Narrativa de V. M. Santa Marta, mayo de 2016.

34 Ibídem. 
Aparece también, la incidencia familiar (Identidad con la profesión materna), en la voz de una de las maestras Psicopedagoga y Doctora en Educación:

"Considero que mi decisión de ser maestra está influenciada por mi madre, ella fue maestra durante 50 años. Recuerdo que cuando era niña, mi madre fue maestra rural y me llevaba con mis hermanas menores a los lugares donde se desplazaba a trabajar, la veía enseñar a distintos niveles de primaria al tiempo (Escuela multigrado/Integrada), ahora sé que es la metodología de Escuela Nueva. Para mí era mágico todo eso". ${ }^{35}$

Cabe destacar que casi todas las maestras afrocolombianas participantes en la investigación manifiestan en sus narrativas un especial interés en llevar a cabo estrategias y actividades relacionadas con la implementación de la Cátedra de Estudios Afrocolombianos; por ello expresan que además de la formación de pregrado, han realizado Cursos, Seminarios, Diplomados, Jornadas relacionadas con su puesta en práctica y con la educación intercultural. Estas modalidades de formación continua han contribuido a que se den cambios en sus enfoques educativos, que afectan, en algunos centros, a todas las áreas; y en otros, en actividades puntuales.

\section{Finalidades}

Ilustración 2. Finalidades para alcanzar el desarrollo profesional

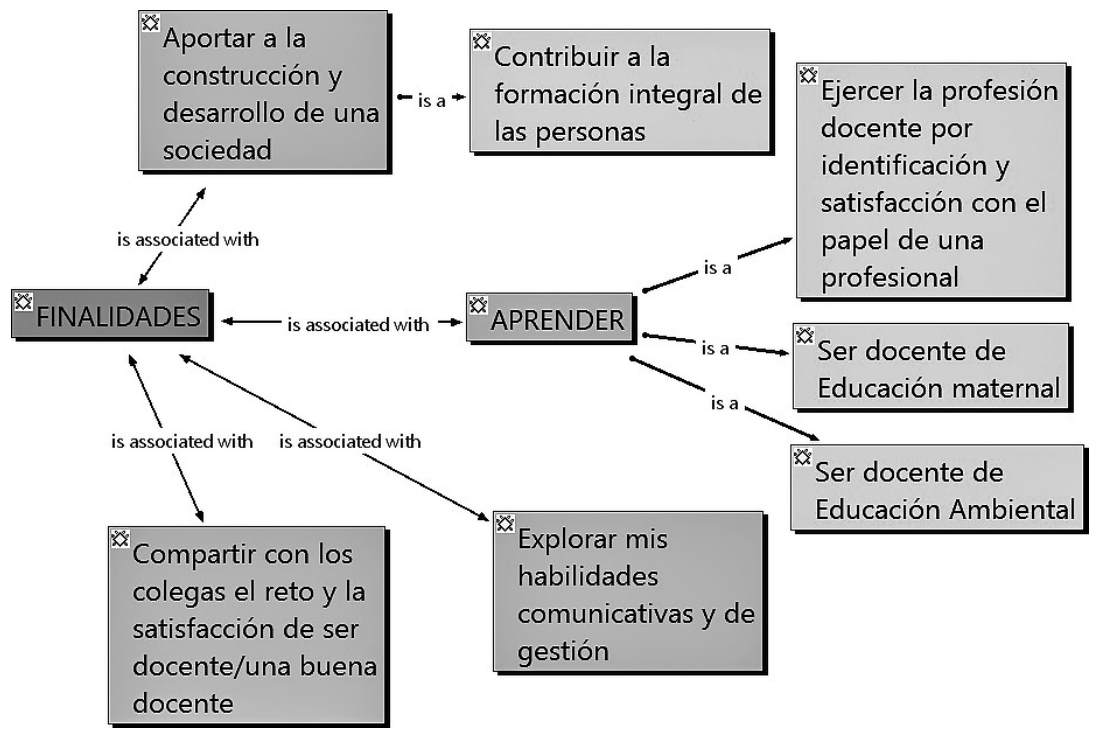

Fuente: elaboración propia mediante el programa Atlas ti.

Las maestras participantes en la investigación manifiestan la relevancia de su trabajo en la construcción de una sociedad igualitaria, dado que pueden contribuir a la formación y desarrollo integral de las personas. Así lo manifestaban:

35 Entrevista a R. R. Santa Marta, noviembre de 2015. 
"al educar ciudadanos con valores, principios y abiertos al mundo"; "despertar el espirítu científico de los estudiantes" "Tengo la oportunidad de apoyar a chicos y chicas con muchas necesidades para ser ciudadanos de bien"36

"Lograr motivar a los estudiantes y contribuir a su formación integral, para superar la vulnerabilidad y deprivación social". ${ }^{37}$

Por otro lado, enfatizan la importancia del trabajo colaborativo en desarrollo de las diferentes actividades, no sólo con los y las otras docentes, también con las familias, como lo expresaban una docente de Juan y Medio y otra de Santa Marta, respectivamente:

"He tratado de que los estudiantes trabajen en equipo y se respeten, se autoreconozcan, que hablen, investiguen, que traigan aportes y saberes desde su familia, rescatando un poco la tradición oral, invitamos a los padres a que cuenten su experiencia tanto desde la parte afro como de la parte wayú". ${ }^{38}$

"A través del proyecto Valoro y aprendo mis raíces afrocolombianas, cuyo objetivo es trabajar la identidad de los niños pertenecientes a este grupo étnico y que conozcan su cultura. Este proyecto se trabaja con la colaboración de los padres de familia". ${ }^{39}$

Las maestras consideran que, en los contextos en que trabajan, de gran diversidad cultural, es importante implementar vías que permitan una comunicación y colaboración fluidas con la comunidad. Reconocen que las familias y comunidades son fuente de conocimiento y aprendizaje, no solo para los alumnos, también para ellas en su labor diaria. 


\section{Desempeño profesional}

Ilustración 3. Desempeño profesional de las maestras afrocolombianas participantes en las narrativas.

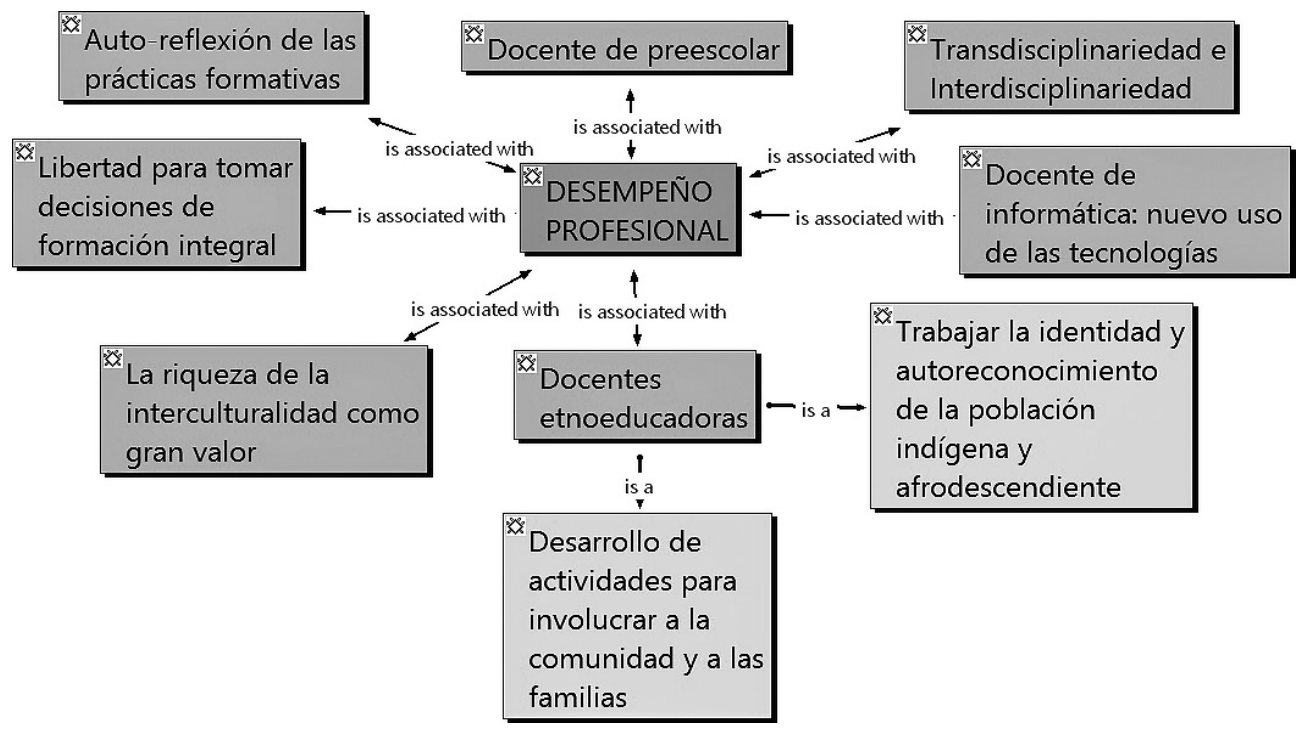

Fuente: elaboración propia mediante el programa Atlas ti.

Las maestras afrodescendientes valoran su desempeño profesional como etnoeducadoras, al implementar estrategias para trabajar la identidad y el auto reconocimiento en la población estudiantil afrodescendientes e indígena, como enfatiza la directora de la Institución Etnoeducativa Sierra Nevada y M. R. Doctora en Ciencias, maestra de Santa Marta:

"Creo que debemos ser muy cuidadosas porque en nuestro lenguaje cotidiano utilizamos muchas expresiones discriminatorias, que se han ido repitiendo de generación en generación y que como decía antes están muy arraigadas en toda la población: blanca, mestiza, indígena y negra. Por eso estamos trabajando mucho la identidad en la población afro, lo hacemos desde pre-escolar." ${ }^{40}$

"Trabajamos sobre todo el conocimiento de cada cultura y del respeto hacia ellos mismos y hacia las otras culturas, partimos del fortalecimiento de la identidad, primero tratando de que se reconozcan desde sus ancestros e historia, cuando hacíamos el diagnóstico de sondeo antes de empezar el trabajo de la Cátedra, encontramos que a pesar de que se ha avanzado en ese nuevo reconocimiento, había muchas personas que se avergonzaban de ser negros". ${ }^{41}$

$40 \quad$ Entrevista a L. S. Juan y Medio, abril de 2015.

41 Entrevista a M. R. Santa Marta, marzo de 2016. 
La docente Ana Rita Serrano, destaca la necesidad de fomentar el conocimiento entre los diferentes grupos étnicos:

"Tenemos a los indígenas de la Sierra Nevada muy cerca, son nuestros vecinos y a la vez nuestros grandes desconocidos, a veces, aunque no es mi caso, tengo la sensación de que pensamos que su historia es algo que no nos compete". ${ }^{42}$

Así mismo, consideran relevante como maestras afrodescendientes realizar un trabajo de sensibilización, que potencie el diálogo entre culturas "Si sobre todo la sensibilización, porque hay mucho desconocimiento como afrocolombianos, ya que los trabajos que se hacen son en sectores deprimidos y en la periferia de la ciudad, en la parte urbana considero que se trabaja poco. Trabajo con los estudiantes desde el respeto y la escucha, considerando el punto de vista del otro". ${ }^{43}$

El análisis de esta parte de las narrativas indica la necesidad de realizar un trabajo alrededor de la identidad en los grupos minoritarios en Colombia, con mayor énfasis en la población afrodescendiente, cuya historia y la manera como poblaron el continente americano, aún pesa y condiciona, en muchos ámbitos, las relaciones con el resto de la población. Se destaca que, dentro de este trabajo, identidad y auto reconocimiento, ocupa un lugar importante la labor educativa desarrollada a nivel de la comunidad.

Por otro lado, se pone de relieve el valor de que todo docente debe tener una óptima preparación ante el reto de trabajar con jóvenes de ambientes y familias vulnerables; así como el trabajo colaborativo, interdisciplinar y transdisciplinar, sobre todo en aquellas Instituciones en que la Cátedra y la Etnoeducación están insertas en el curriculum.

42 Narrativa de A. R. Serrano. Santa Marta, mayo de 2016.

43 Entrevista a M. R., Santa Marta marzo de 2016. 


\section{Métodos y Estrategias}

Ilustración 4. Métodos y estrategias utilizadas por las maestras en su práctica educativa.

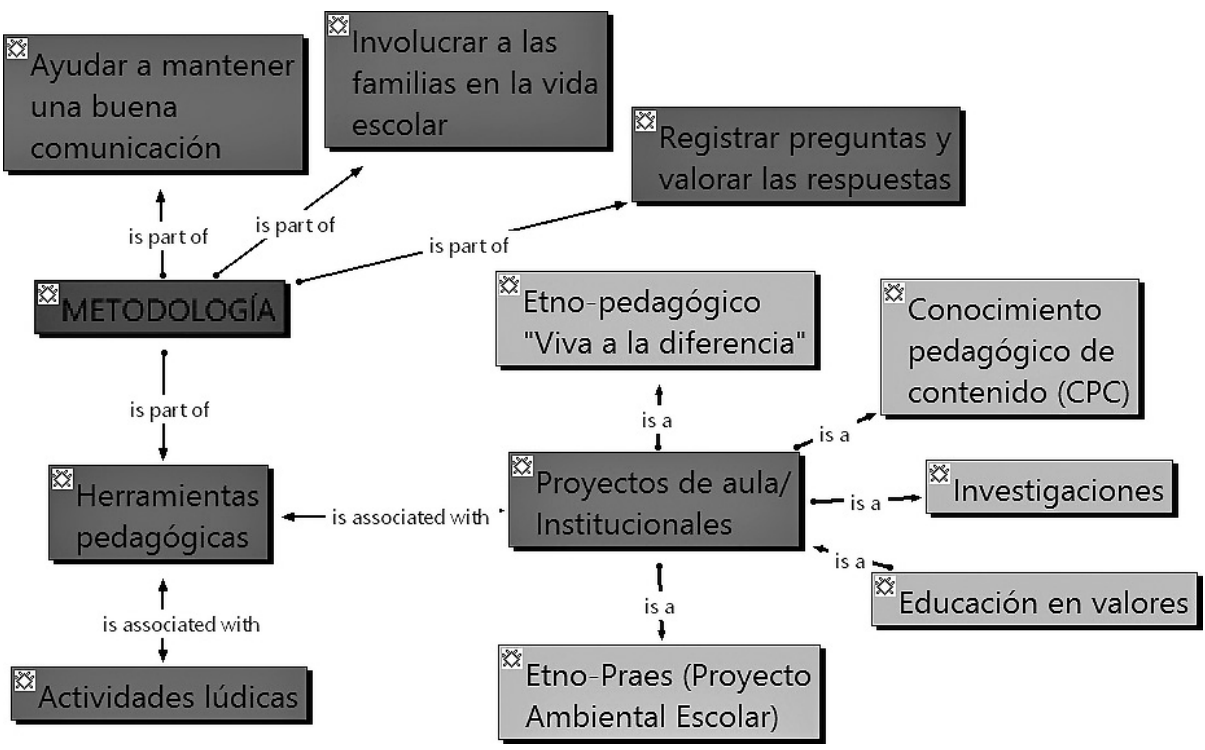

Fuente elaboración propia mediante el programa Atlas ti.

Las voces de las maestras abundan en la necesidad de descubrir, a través de la comunicación, a las personas, naturaleza, comunidades, colores "relacionándose desde el respeto, amor solidaridad, igualdad, teniendo en cuenta las normas básicas de convivencia" ${ }^{44}$

Así mismo, J. C., enfatiza la importancia de realizar un registro de preguntas, sobre todo de los alumnos más pequeños y valorar sus respuestas:

"¿Por qué ese niño tiene tantos colores en la cara y en su cuerpo?"

"Todos los niños somos iguales",

"Los niños negros no pueden cambiar de color porque son negros". 45

Considera que a edades tempranas es importante la atención a este tipo de inquietudes que deben resolverse a través de conversatorios y lluvia de ideas y a la vez "emerger nuevas tareas y promover un aprendizaje significativo, globalizador y activo". 46

Una herramienta pedagógica que las docentes refieren utilizar con frecuencia son las actividades lúdicas, ya sea a través de juegos, dramatizaciones, canciones, mitos, leyendas, etc.

\footnotetext{
44 Entrevista a R. R. Santa Marta, noviembre de 2015.

45 Narrativa de J. C. quien ha llevado a cabo con gran éxito el proyecto "Viva la diferencia" con alumnos de preescolar en su Institución educativa. Santa Marta, abril de 2016.

46 Ibídem.
} 
Entre las herramientas pedagógicas, además de la experiencia directa, entrevistas, registros de información, juegos, dramatizaciones, actividades grupales y recursos literarios; las maestras participantes en la investigación consideran importante el trabajo a través de proyectos institucionales y de aula:

"Estamos haciendo unos proyectos sobre las costumbres de los kogi, los wiwas y los wayú, en qué se parecen y en qué se diferencian. Es un trabajo de investigación muy amplio y muy interesante, ya que tendemos a creer que todos son iguales y hay también particularidades en cada una de las culturas, porque de pronto los tratamos de la misma forma y nos equivocamos"

Algunas de las maestras narran en sus historias, el diseño de Proyectos de investigación relacionados con los diferentes grupos étnicos, sus costumbres, formas de vida, características, etc. En las investigaciones llevadas a cabo se implica a todos los alumnos y a las familias.

"Los estudiantes investigaron sobre la historia del pueblo, de nuestros personajes, del significado de nuestros ritos y costumbres, de dónde vienen. Todo eso lo plasmamos en carteleras, dibujos; luego hicimos lo mismo, pero con personajes afro de fuera de nuestro contexto". 48

Imagen 2. Presentación del ETNOPRAES a la comunidad educativa

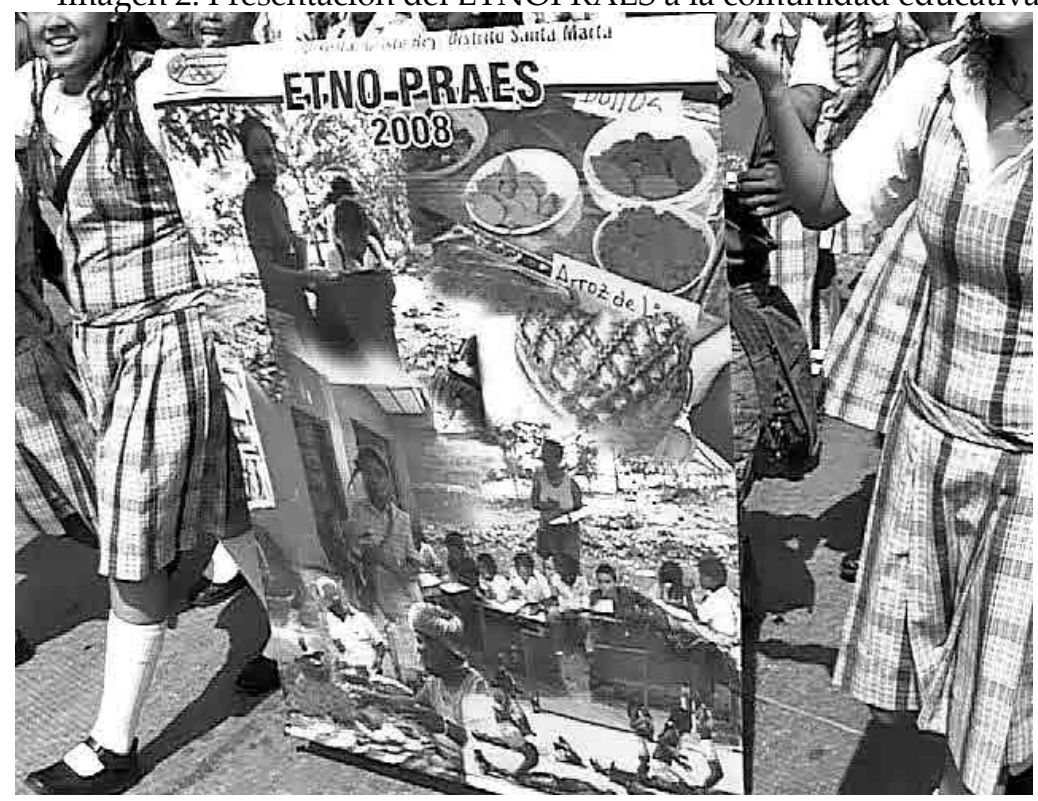

Fuente: Meri Ruiz. Maestra participante en la investigación. Santa Marta, marzo de 2016.

47 Entrevista a I. S. Juan y Medio, abril de 2015.

48 Entrevista a las maestras I. S., B. S. y L. S. Juan y Medio, abril de 2015. 
“Elaboramos el ETNOPRAES, un Proyecto Ambiental Escolar Afro para resaltar las relaciones étnicas que se dan en esta comunidad educativa y visibilizar los aportes de la etnia Afro caribe al desarrollo socioeconómico y cultural de la región." ${ }^{49}$

En esta estrategia se unen los Lineamientos de Educación Ambiental, la propuesta de la Ley 115/94 en su Art. 14, que dice que Los establecimientos educativos Privados y Públicos, están obligados a cumplir con proyectos Pedagógicos Transversales, Ley General de Educación y el Decreto 1122 de 1998, que hace obligatoria la cátedra de estudios afrocolombianos, que comprende un conjunto de temas, problemas y actividades pedagógicas relativos a la cultura propia de las comunidades negras, y se desarrolla como parte integral de los procesos curriculares de ciencias sociales, historia, geografía, constitución política y democracia.

Las maestras de las Instituciones donde se han desarrollado este tipo de proyectos e investigaciones, subrayan la importancia de involucrar en su desarrollo a la comunidad y las familias, especialmente a los mayores, dado que son los portadores y conocedores de la historia y costumbres.

En el proyecto titulado "Conocimiento pedagógico de contenido sobre diversidad en las prácticas pedagógicas del futuro docente de ciencias naturales: Un estudio de caso", se trata de aprovechar lo desbordante de la curiosidad de los estudiantes, sus inquietudes, la diversidad cultural y los recursos del medio natural, como lo expresa una de las docentes afrocolombianas del contexto rural "se trata de integrar todas las áreas de conocimiento y articular en la asignatura todo lo que han aprendido" 50

Reconocen que muchos de los estudiantes y sus familias, afros, indígenas y mestizos, han vivido situaciones de desarraigo que requieren una atención especial, que tratan de brindar las docentes, en muchas ocasiones con ayuda de otros especialistas. Sin duda la educación en valores, el fortalecimiento de la autoestima en este tipo de población es una necesidad de la que los docentes manifestaban lo siguiente:

"He participado en talleres sobre educación en valores, creo que es fundamental cuando se trabaja en el contexto de mi escuela". ${ }^{51}$

"Hemos hecho varios talleres sobre formación en valores, con la ayuda de la psicóloga del colegio". 52

"Realizamos varios talleres sobre educación en valores, porque es realmente importante que los alumnos aprendan a respetarse". ${ }^{53}$

49 Entrevista a M. R. Santa Marta, marzo de 2016.

50 Entrevista a I. S. Juan y Medio, abril de 2015.

51 Narrativa de V. M. Santa Marta, mayo de 2016.

52 Narrativa de J. C. Santa Marta, abril de 2016.

53 Narrativa de A. R. S. Santa Marta, mayo de 2016. 
En todas las narrativas, se enfatiza la necesidad de trabajar proyectos de educación en valores, talleres de autoestima y fomentar la formación para el trabajo, dada la situación de precariedad socioeconómica de los contextos en los que se desempeñan las maestras afrocolombianas participantes en la investigación.

\section{CONCLUSIÓN}

Con respecto al contexto de su formación, las maestras en la investigación presentan diversos perfiles, la totalidad de las participantes son licenciadas con estudios de postgrado y han realizado diversas actividades formativas de formación permanente.

Por otro lado, se ha tratado de abordar tanto el contexto urbano, como el rural en las narrativas recogidas, lo que ha aportado distintos puntos de vista sobre el trabajo y las prácticas docentes realizadas. Sin duda en el contexto rural, las maestras afrodescendientes, desarrollan su docencia desde una perspectiva multicultural implementando estrategias de marcado enfoque intercultural, propiciando el diálogo, conocimiento y reconocimiento de los distintos grupos étnicos presentes en las instituciones educativas (Ruiz, 2013 ${ }^{54}$, Ruiz y Medina, $2014^{55}$, Avoro y Ruiz, 2016 ${ }^{56}$ ).

En el contexto urbano, las maestras afrocolombianas implicadas en la investigación, llevan a cabo actividades diversas orientadas al desarrollo de la Cátedra de Estudios Afrocolombianos, a través de estrategias que abordan el reconocimiento de la identidad, así como la visibilización y reconocimiento como afrodescendiente.

Son significativas las voces de las maestras al afirmar en sus narrativas, que tienen en cuenta en su diseño educativo todos los aspectos relacionados con los contextos social y cultural, y además que tratan de resaltar en todos los contenidos las costumbres y saberes de cada cultura, tanto en los proyectos que realizan como en los contenidos de las asignaturas.

Las maestras son conscientes de la riqueza que representa la incorporación de personas representativas de las comunidades, tanto indígenas como afrodescendientes en la organización de la vida escolar, para el fortalecimiento de los proyectos educativos y para fomentar el conocimiento y el respeto hacia los grupos étnicos minoritarios en toda la comunidad educativa.

54 Adiela, Ruiz Cabezas. "Estudio Comparativo sobre los métodos utilizados en educación intercultural en la población negra e indígena de Santa Marta y Palenque y la población de Málaga” (Tesis Doctorado en Universidad Nacional de Educación a Distancia, 2013).

55 Adiela Ruiz Cabezas, Antonio Medina Rivilla. "Modelo didáctico intercultural en el contexto afrocolombiano: la etnoeducación y la cátedra de estudios afrocolombianos". Indivisa: Boletín de Estudios e Investigación, 14 (2014): 6-29.

$56 \mathrm{M}^{\mathrm{a}}$ Teresa Avoro Nguema Ebana y Adiela Ruiz Cabezas "Necesidades de formación del profesorado en la competencia intercultural en Malabo, Guinea Ecuatorial” Indivisa: Boletín de Estudios e Investigación, 16 (2016): 105-126. 
En cuanto a las finalidades para alcanzar el desarrollo profesional, las maestras consideran que lo fundamental es "aprender a ser docente" 57 , dentro de sus respectivos ámbitos de conocimiento y sobre todo a través de su práctica educativa, compartiendo con los colegas el reto y la satisfacción de ser una buena docente y como lo expresaba alguna de ellas, "descubrir el toque mágico de ser docente". 58

En lo concerniente al desempeño profesional, destacamos algún dilema profesional en dos de las docentes participantes al expresar "yo nunca pensé ser profesora" ${ }^{29}$, sin embargo, una de ellas manifiesta que a lo largo de su práctica y experiencias ha descubierto que "ser maestro es ser un todo, es enamorarse de lo que se hace"60; palabras que demuestran el nivel de compromiso de estas maestras afrocolombianas.

Todas las maestras enfatizan la importancia de trabajar en proyectos que den a los niños la oportunidad de aprender a ser diferentes desde todos los puntos de vista, especialmente desde la diversidad cultural. En todas las narrativas se expresa la convicción de ligar el desempeño profesional a la riqueza cultural como un valor, trabajando y llevando a la práctica diversas estrategias y metodologías que ayuden a mantener una buena comunicación, a través de diversas herramientas pedagógicas como los proyectos de aula e institucionales que involucren a toda la comunidad educativa: docentes y familias.

Las maestras subrayan la necesidad trabajar de forma creativa y lúdica, utilizando los mitos, leyendas y cuentos transmitidos de generación en generación, como una herramienta para trabajar la identidad y ayudar a superar y /o prevenir los conflictos entre las diferentes culturas presentes en las aulas.

En cuanto a la temporalización de las experiencias formativas que las han marcado en su práctica educativa, prácticamente todas manifiestan que se trata del conocimiento adquirido para trabajar en y desde la implementación de la Cátedra de Estudios Afrocolombianos y del enfoque intercultural. Formación que ha sido recibida en los cursos, jornadas, seminarios y diplomado que han realizado.

Finalmente, en este artículo se ha tratado de ligar la metodología narrativa a la emergencia y valoración de experiencias formativas previas que enriquecen las nuevas narrativas y sitúan a cada educadora como la protagonista de su propia formación, compartiendo experiencias de proyección en las nuevas formas de avanzar y comprender la diversidad de acciones y escenarios formativos de cada implicado en las actuaciones innovadoras de las maestras.

57 Entrevista a R. R. Santa Marta, noviembre de 2015.

58 Narrativa de V. M. Santa Marta, mayo de 2016.

59 Narrativa de J. C. Santa Marta, abril de 2016. Entrevista a R. R. Santa Marta, noviembre de 2015.

60 Narrativa de J. C. Santa Marta, abril de 2016. 


\section{FUENTES}

Entrevistas realizadas por Adiela Ruiz Cabezas en 2015 y 2016.

Entrevista a Nuria Padilla. Santa Marta, febrero de 2015.

Entrevista a Lenneys Polo. Santa Marta, marzo de 2015.

Entrevista a Berlys Sierra. Juan y Medio, abril de 2015.

Entrevista a Irina Sarmiento. Juan y Medio, abril de 2015.

Entrevista a Ledys Sarmiento. Juan y Medio, abril de 2015.

Entrevista a Rubiela Ruiz. Santa Marta, noviembre de 2015.

Entrevista a Meri Ruiz. Santa Marta, marzo de 2016.

Narrativas recogidas por Adiela Ruiz Cabezas en 2016.

Narrativa de Julia Cataño. Santa Marta, abril de 2016.

Narrativa de Ana Rita Serrano Santa Marta, mayo de 2016.

Narrativa de Victoria Mejía. Santa Marta, mayo de 2016.

\section{REFERENCIAS}

Avoro Nguema Ebana, Mํㅗㄹ Teresa y Ruiz Cabezas, Adiela. “Necesidades de formación del profesorado en la competencia intercultural en Malabo, Guinea Ecuatorial" Indivisa: Boletín de Estudios e Investigación, 16 (2016): 105-126.

Bochner, Arthur, Ellis, C., Sava, I., Nuutinen, K., Mäkelä, M., Ylönen, M. E. y Sclater, S. D. "The arts and narrative research: Art as inquiry". Qualitative inquiry 9, No. 4 (2003): 505-624.

Cañón Flórez, Lilia; Monroy González, Miriam Esperanza y Salcedo Casallas, Javier Ricardo. “Experiencia pedagógica y voz multicultural: Pasos y palabras en historia de vida de maestra afrodescendiente". Revista Historia de la Educación Latinoamericana 18, No. 27 (2016):167-190.

Cazden B. Courtney. "Las aulas como espacios híbridos para el encuentro de las mentes." En Aprendizaje y contexto: contribuciones para un debate, editado por Nora Elichiry (Buenos Aires: Ediciones Manantial, 2010).

Chase, Susan E. "Narrative Inquiry. Still a field in the making". En The Sage Handbook of Qualitative Research, editado por Norman Denzin y Yvonne S. Lincoln. (California: Thousand Oaks, 2011), 421-434.

Connelly, F.M. y Clandinin, D. J. “Stories of experience and narrative inquiry”. Educational Researcher 19, No. 5 (1990): 2-14.

Connelly, F. M. y Clandinin, D. J. "Narrative inquiry". En Complementary methods for research in education, editado por JL Green, G. Camilli. PB Elmore Eds., 2006, 477487.

Creswell, J. W. Educational research: Planning, conducting and evaluating quantitative and qualitative research. Boston, US: Pearson, 2012.

Creswell, J. W. Qualitative inquiry $\mathcal{E}$ research design: Choosing among five approaches. Thousand Oaks, US: SAGE, 2007.

Denzin, Norman. Interpretative autoethnography. (California: Thousand Oaks, 2014). 
Goodson, Ivor, Loveless, Avril y Stephens, David. (Eds.), Explorations in narrative research. (Rotterdam: Sense Publishers, 2012).

Huber, Gunter. "investigación en la comunicación didáctica”. En Las Fronteras de la Investigación Didáctica, editado por Antonio Medina, Agustín de la Herrán y Ma Concepción Domínguez. (Madrid: Editorial UNED, eBook 2014), 73-114.

Huber, Gunter. "Aprendizaje activo y metodologías educativas". Revista de Educación Número extraordinario (2008): 59-81.

Leithwood, Kenneth A. y Hallinger Philip eds. Second international handbook of educational leadership and administration. (Springer Science \& Business Media, 2012).

Leithwood, Kennet A., Jantzi, Doris y Mcelheron-Hopkins, Charrin. “The development and testing of a school improvement model". School effectiveness and school improvement 17, No. 4 (2006): 441-464.

Lima Jardilino, José Rubén y Michaltchuk, Ivana Elena. "La maestra en la escuela rural itinerante: Una experiencia de educación rural en la región sur de Brasil". En La maestra rural en Iberoamérica, eds. Diana Elvira Soto, Justo Cuño y Oscar Hugo López (Tunja: Ed. FUDESA, 2015), 271-299.

Medina Domínguez, María y Medina Rivilla, Antonio. “Modelos didácticos y métodos para el desarrollo del conocimiento y la cultura innovadora de las instituciones educativas". En Innovación de la Educación y de la Docencia 2da. Ed, editado por Antonio Medina Rivilla (Madrid: Ramón Areces, 2015), 29-63.

Medina Rivilla, Antonio (coord.). Innovación de la Educación y de la Docencia 2da. Ed. (Madrid: Ramón Areces, 2015).

Medina Rivilla, Antonio y Domínguez Garrido, Ma Concepción. “Modelos didácticotecnológicos para la innovación educativa". En Recursos tecnológicos en contextos educativos editado por Ma. Luz Cacheiro, Cristina Sánchez y Jesús Manuel González (Madrid: UNED, 2015), 27-53.

Medina Rivilla, Antonio. "La investigación como base del conocimiento didáctico y de la innovación de la enseñanza". En Las Fronteras de la Investigación Didáctica, editado por Antonio Medina, Agustín de la Herrán y Ma Concepción Domínguez. (Madrid: Editorial UNED, eBook 2014), 23-72.

Medina Rivilla, Antonio. "La biografía perspectiva de la formación del profesorado: limitaciones y posibilidades". En. Historias de vida e investigación etnográfica, editado por Emilio López-Barajas Zayas y Magdalena Montoya Sáenz (Madrid: UNED, 1996).

Medina Rivilla, Antonio y Domínguez Garrido, Maㅡ Concepción “Constructing teacher's practical knowledge through analysis of Geography Teacher's Discourse". En Experimental Research on Teaching and Learning, editado por Olechowski y KhanSrik (Frankfurt: Peter Lang 1995), 95-117.

Ruiz Cabezas, Adiela; Medina Rivilla, Antonio. "Modelo didáctico intercultural en el contexto afrocolombiano: la etnoeducación y la cátedra de estudios afrocolombianos". Indivisa: Boletín de Estudios e Investigación 14, (2014): 6-29.

Ruiz Cabezas, Adiela. "Estudio Comparativo sobre los métodos utilizados en educación intercultural en la población negra e indígena de Santa Marta y Palenque y la población de Málaga". Tesis Doctorado en Universidad Nacional de Educación a DistanciaUNED, 2013. 
Soto Arango, Diana Elvira; Avoro Nguema, Maㅡ Teresa; Ruiz Cabezas, Adiela y Medina Rivilla, Antonio. (2016). Formación de docentes en Guinea Ecuatorial. Historias de vida de maestras en prospectiva al 2020. Revista Historia de la Educación Lationoamericana 18, No. 27, (2016): 67-94.

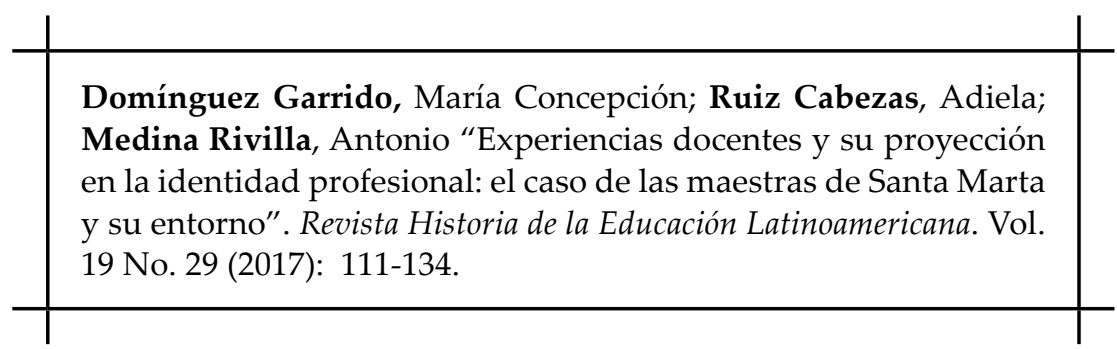

